\title{
Zigbee-based Intelligent Home Furnishing
}

\author{
Huang Jinhui, Xue ZiYu, Xu Xu and Tian Siyang \\ Computer and Information Engineering, Beijing Technology and Business \\ University \\ huangjh@th.btbu.edu.cn
}

\begin{abstract}
With the constantly emerging of the high-tech, Household life of intelligent, network, digitization has become a research direction in the field of Internet of things. Against this background, this paper puts forward a set of intelligent household iot system based on ZigBee architecture design. The design scheme can be connected to various devices in the family, In order to realize people and equipment, equipment and equipment between "communication", Greatly convenient people's home life. Because the ZigBee module has many advantages such as save electricity, small volume, low cost .Based on ZigBee smart home system has great research value, and is a good way to promote the speed of the Internet of things into our life, let people know well to the role of the Internet of things and it is convenient for our life.
\end{abstract}

Key words : Intelligent Home Furnishing ; Internet of Things ; Zigbee

\section{Introduction}

As 3C (Computer Consumer Electronic Communication) technology, network technology and furniture intelligent technology are constantly promoted, the future home, intelligentized, networked and digitized, gradually becomes the important development subject of the internet of things nowadays. This paper introduces a kind of Zigbee technology based system architecture of smart home internet of things. Integrating Zigbee wireless module, sensor technology and network technology, the system reflects the embodying mode of smart home.

\section{Comparative Study of the Existing Technology}

\subsection{The Existing Technology and Scheme $[1,2,3]$}

With the constant development of communication technology, all kinds of short-range wireless communication technologies are being innovated continuously. The current available technologies include Bluetooth technology, WI-FI technology and Zigbee technology.

Bluetooth technology is created by Ericsson initially. On May 20th, 1999, Sony Ericsson, IBM, Intel, Nokia, Toshiba and other leaders of the industry established the Special Interest Group of Bluetooth and formulated the standards of Bluetooth technology. In 1998, Ericsson wished the standards of wireless communication technology could be unified and name it as "Bluetooth". Blue tooth technology is committed to enable all mobile or immobile devices supporting the technology to form network association conveniently, conduct voice and data communication in the space of $10 \sim 100 \mathrm{~m}$.

WiFi[4] (Wireless Fidelity), namely IEEE802 . 11 protocol, is a kinds of short-range wireless transmission technology capable to support the internet accessed radio signal 
within a few hundred feet. The first version of WiFi was published in 1997, in which MAC and PHY are defined.

The basic network structure and the basic transmission medium of Wireless Local Area Network are stipulated and the features of PHY and MAC are specified. The PHY is adopted with infrared, DSSS or FSSS technology. In 1999, the standards of IEEE 802.

11a and IEEE 802 . $11 \mathrm{~g}$ are added. Its maximum transmission rate could reach $54 \mathrm{Mb} / \mathrm{s}$ and it is widely capable to support data, image, voice and multimedia and other businesses.

Zigbee[5], the pronoun of IEEE802 . 15 . 4 protocol, is derived from the zig dancing of bees. As bees rely on flying and zig "dancing" to tell companions where pollen is, in other words, bees rely on this way to form the communication network in the colony. It is characterized by close range, low complexity, self-organizing, low-power dissipation and low cost, mainly suitable for fields of automatic control and remote control and could be embedded in various equipments.

Table 1 Comparison Between Bluetooth, WI-FI and Zigbee Wireless Communication Technologies

\begin{tabular}{llll}
\hline Name/Parameter & Bluetooth & Wi-Fi & Zigbee \\
\hline Operating Frequency Range & $24 \mathrm{FHz}$ & $24 / 52 \mathrm{GHz}$ & $\begin{array}{l}868 \mathrm{MHz} / 915 \mathrm{MHz} / \\
24 \mathrm{GHz}\end{array}$ \\
Transmission Rate $/(\mathrm{b} / \mathrm{s})$ & $1 \mathrm{M} / 3 \mathrm{M}$ & $11-54 \mathrm{M}$ & $20 \mathrm{k} / 40 \mathrm{k} / 250 \mathrm{k}$ \\
Transmission Distance/m & 10 & $15-100$ & $10-100$ \\
Transmitted Power $/ \mathrm{mW}$ & $1-100$ & 100 & $1-3$ \\
Maximum Link Points & 7 & 32 & $255 / 65535$ \\
Security & $128 \mathrm{bit}$ secret & SSID & $\begin{array}{l}\text { AES-128 } \\
\text { encryption }\end{array}$ \\
Response Time & key & & $30 \mathrm{~ms}$ \\
Battery Life & $3 \mathrm{~s}$ & $10 \mathrm{~s}$ & $1-2 \mathrm{a}$ \\
\hline
\end{tabular}

\subsection{Shortcomings of the Existing Technologies $[6,7]$}

Although the Bluetooth technology under the standard 4.0 avoids the bottleneck of data transmission, forms low-power design, and the communication distance reaches the range of $2.4 \mathrm{G}$ wireless signal, its defects are still obvious and it's not suitable for smart home design. Its defects are mainly shown in the following three aspects:

1. Inadequate anti-interference capability: the networking scale under the new standards is generally no more than 300 and Bluetooth is easy to be interfered.

2. Poor self-organized networking: for Bluetooth, one network port corresponds to eight ports at the most and it's impossible to extend, normally, Bluetooth is not used as the application foundation of the internet of things.

3. Inadequate security: as Bluetooth technology is not equipped with network selfhealing function, secret divulging events happen to Bluetooth commonly[9]. 
WI-FI also has similar shortcomings, for example, WIFI roughly corresponds to about 20-30 ports of commercial network ports, for families, one network port corresponds to ten ports. Therefore, if building the 30-port home automation, it needs three suits of different network ports, which is obviously unbearable for ordinary families.

\section{Feasibility of Selecting Zigbee Module}

The smart home system proposed in this paper is based on Zigbee wireless technology. In order to make it convenient to discussing the feasibility of such smart home system, some basic concepts of Zigbee are introduced in the following firstly.

\subsection{Zigbee Wireless Network [10]}

Zigbee is a kind of short-range, low-power dissipation wireless communication technology characterized by short distance, low complexity, low-power dissipation, low data rate and low cost. Compared with other wireless communication technology, Zigbee has the following technological advantages:

(1) Low-power dissipation. In low-power dissipation standby mode, two No.5 dry batteries could support one mode to work for $6 \sim 24$ months and even longer. It's not convenient for smart home to change batteries frequently.

(2) Low cost. Low cost is the premise to guarantee family installation. Through largerange simplification protocol (less than 1/10 of that of Bluetooth), Zigbee module lowers the requirements for communication controller, bases on predictive parsing, measures and calculates with the 8-bit microcontroller of 8051 , full-function main nodes need $32 \mathrm{~KB}$ code and sub-function nodes need as little as $4 \mathrm{~KB}$ code.

(3) Low rate. Zigbee works at the low rate of $20 \sim 250 \mathrm{kbps}$ and respectively provides the original data throughput rates of $250 \mathrm{kbps}(2.4 \mathrm{GHz}) 、 40 \mathrm{kbps}(915 \mathrm{MHz})$ and $20 \mathrm{kbps}(868 \mathrm{MHz})$ to meet the application needs of low-velocity data transmission [11].

(4) Short time delay. Zigbee responses fast, general nodes only need $30 \mathrm{~ms}$ to connect with and access the network and only need $15 \mathrm{~ms}$ from sleeping to working, saving more electric energy. Comparatively, WiFi needs $3 \mathrm{~s}$.

(5) High security. Zigbee provides the three-level security mode, including no-security setting, ACL, preventing illegal access to data and adopting the symmetric cryptography of advanced encryption standard (AES 128), and determines the security attribute with flexibility.

\subsection{The Positioning Accuracy of Zigbee Module}

Zigbee adopts RSSI technology for positioning. Positioning security could ensure the controllability and security of the whole system. RSSI ( Received Signal Strength Indicator ) is the strength indicator of received signals.

RSSI is realized after backward channel baseband receiving the filter. In order to obtain the features of reverse signals, the following is managed in the concrete realization of RSSI: baseband IQ power integration is done within 104us to get the RSSI instantaneous value, namely RSSI (instantaneous) $=$ sum $\left(I^{\wedge} 2+Q^{\wedge} 2\right)$; then average the 8192 RSSI instantaneous values within about 1 second to get the average value of RSSI, namely RSSI (average) =sum (RSSI(instantaneous) )/8192, meanwhile offer the maximum RSSI instantaneous value and the ratio of RSSI instantaneous value greater than a certain threshold (the number of RSSI instantaneous value greater than a certain threshold/8192).As RSSI is got through power integration in numeric field and then back- 
pushing to antenna port, the inconformity of backward channel signal transmission characteristic would affect the accuracy of RSSI.

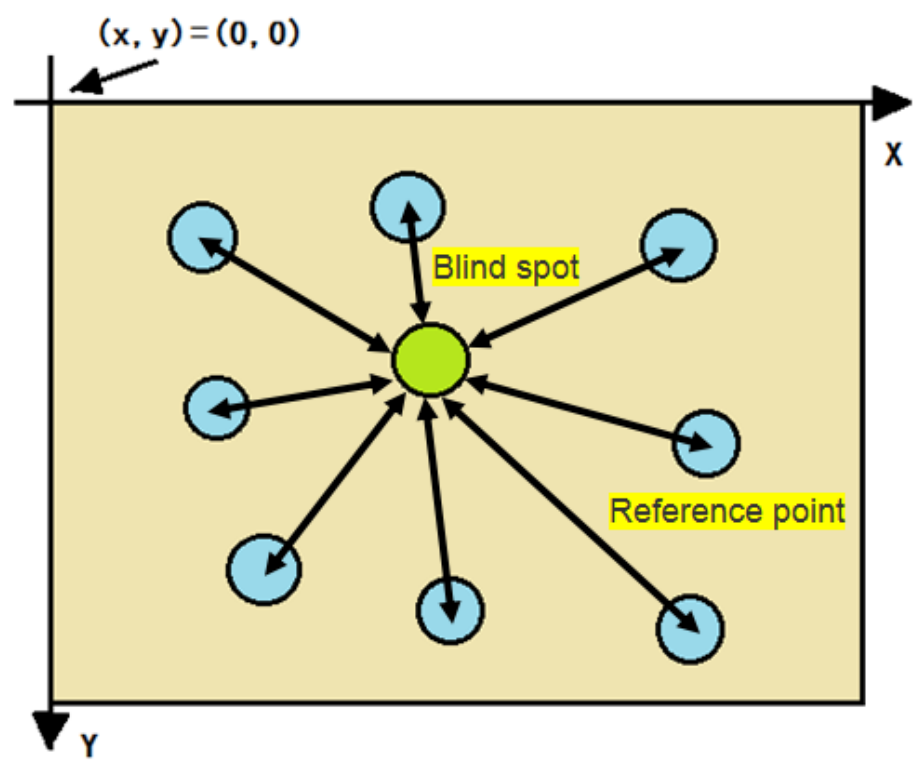

Figure 1. RSSI Positioning Principle Based Diagram

Figure 2 is the RSSI positioning algorithm based principle diagram.

Received signal strength is the function of transmission power and transmission distance (the sender-receiver distance). The received signal strength will decrease progressively along with the increased distance according to the following equation.

$R S S I=-\left(\operatorname{lOnlog}_{10} d+A\right)$

In the equation:

$\mathrm{n}$ : signal propagation constant, also called propagation coefficient.

d : the distance to Zigbee knot.

A : the signal strength at $1 \mathrm{~m}$ to Zigbee knot.

Therefore, if $\mathrm{n}$ and A values are measured, the distance of blind spot relative to each reference point could be worked out based on RSSI value and the coordinate of blind point could be calculated according to triangulation.

In smart home system, each module under Zigbee coordinator is sufficient to cover the whole room and ensure the accuracy of transmission position, the internet is used to transmit between resident rooms and the community server to ensure the long-range transmission and security of information.

\section{The Realization of Smart Home System}

Based on above shortcomings of wireless transmission equipment, this paper proposes to build smart home system by using Zigbee [4] technology. 


\subsection{The Introduction of Smart Home System}

Smart home system transmits monitoring information into Zigbee knot through a sensor and then Zigbee coordinator controls and coordinates. Home gateway controls the condition of the whole smart home coordinator, if confronted with emergency, it could send information to the property management office through the switch and contact residents by mobile equipment at the same time.

Residents could also control electric switches in their houses with mobile equipments and the back-transmitting process will not be given unnecessary details.

Smart home system brings our life infinite convenience. Residents could monitor their houses at work and on journey, as well as turn on or turn off electrical equipments in their houses, greatly improving the security and convenience of housing and accommodation [12].

\subsection{Basic Idea of Smart Home System}

The basic idea and scheme of smart home system is: design two networks which are mutually independent and closely related and build a positioning and sensing network covering the whole home area.

\subsubsection{Zigbee Based Wireless Sensor Network}

The first is Zigbee based wireless sensor network, which is constituted by Zigbee wireless communication module with detecting and transmitting functions, Zigbee coordinator and home gateway with the function of Internet gateway. Zigbee module is installed on house needed position, sends the real-time information sent by the sensor to Zigbee coordinator, and the Zigbee coordinator will collect the information to home gateway[13].

Located on the top of ZigBee wireless network, ZigBee coordinator assumes the responsibilities of building, maintenance and management of the network. ZigBee is equipped with powerful networking capacity, could predominate more than 65,000 nodes at the most theoretically and meet the detective requirements of all kinds of home environmental parameters.

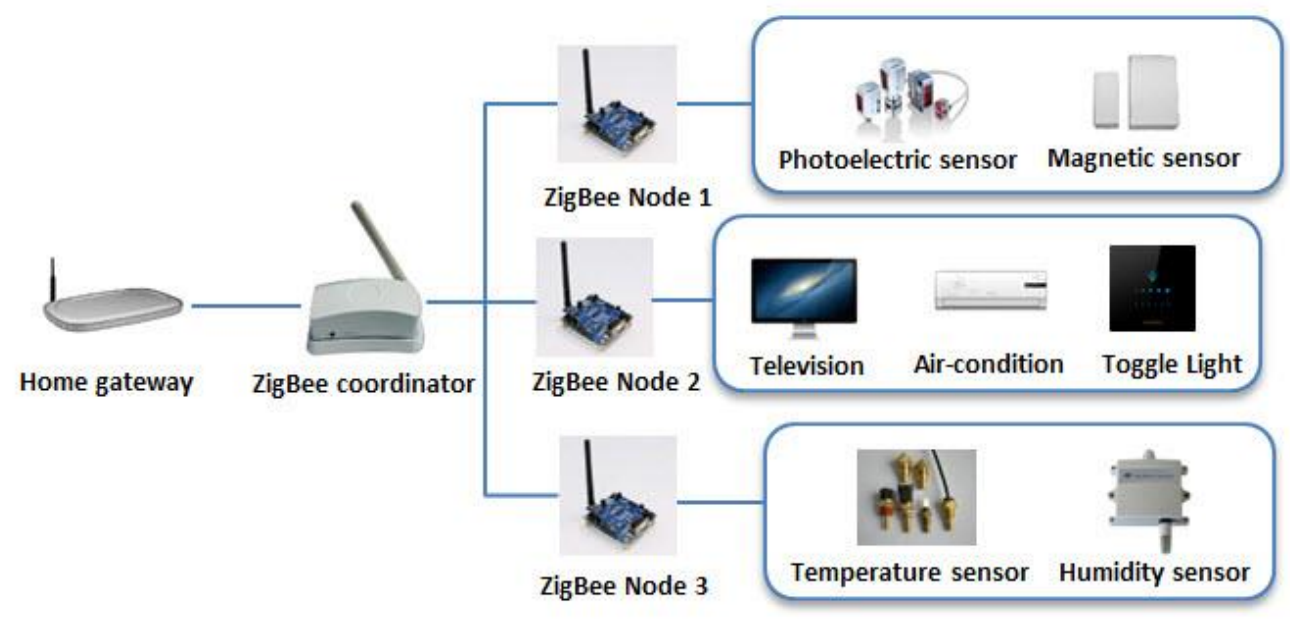

Figure 2. Diagram of Zigbee Based Wireless Sensor Network

\subsubsection{Internet Based Application Network}

The second is the internet based application network, which computes the information collected by the home gateway and stores it in database[14]. In case of emergency, the 
home gateway sends signals to the service provider (property of the community) through the switch in the community and the service provider could position the resident according to where the signal is sent and then proceed the follow-up work.

The two-way reception of home gateway could send the residential security condition to the resident interconnected equipment, meanwhile receive the feedback information of residents, take the gateway as the core and realize the control of household electric appliances, electric lights and relative controlled equipments.

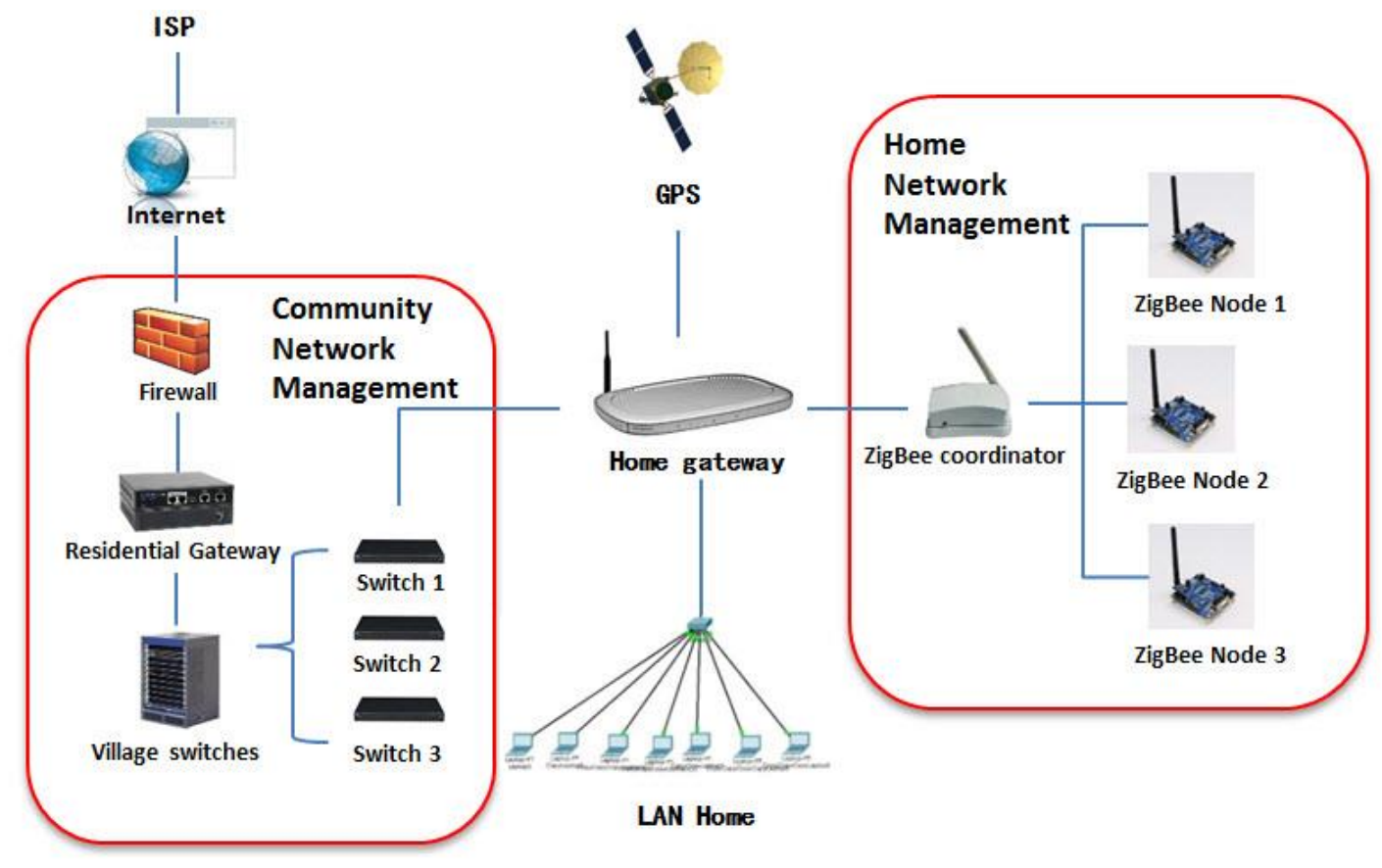

Figure 3. Internet Based Application Network Diagram

\subsection{Description of Smart Home System [15, 16]}

In order to realize the new-type service mode, sensing network is deployed in the specified social area (such as a community) to form a position sensing network firstly. Secondly, home gateway and relative Zigbee sensing network are installed in the house of residents in the community.

Smart home internet of things system interconnects all kinds of household equipments to achieve the "communication" between people and equipment and equipments. For example, gas leakage happens in the kitchen, when the gas sensor monitors that, it will "tell" the gas valve controller the information of gas leakage through ZigBee wireless communication module and makes it close the gas valve; meanwhile, it also "asks" exhaust fan controller to turn on the exhaust fan to ventilate the kitchen. Let's take another example, when the house is on fire, the home internal smoke alarm system will be started timely to inform the property managing center or service provider via internet, mobile phone or telephone network platform to eliminate the dangerous case timely and inform the user working the company or on vocation in another place via messages at the same time. The former hypothesis is usually realized in the home internal ZigBee wireless network and the gateway is needed to realize the later hypothesis that accessing the household equipment via mobile phone or internet and getting to know the equipment running status. Gateway is the intermediary connecting different networks and realizing the interconnection and intercommunication between different networks. In the system of 
smart home internet of things, gateway is the core and it connects the home internal local area network of computer, ZigBee wireless network, GPS network, PSTN network, etc..

\section{Conclusion}

The technology of internet of things develops swiftly and violently unprecedentedly. In China, the concept of internet of things is gradually being known and accepted by people. At the same time of bringing convenience to people's life, home intelligent technology is gradually being deepened and promoted. It is believed that with the national preferential macro-policy to the internet of things market, the future home is that of the internet of things and the future world is that of the internet of things.

\section{References}

[1] F. Yun, "An Introduction to Internet of Things", (2013), pp. 16-26.

[2] S. Subin, "The Conceptual Model and Architecture of Internet of Things", Journal of Nanjing University of Posts and Telecommunications (Natural Science Edition), (2010), p. 15.

[3] L. L. Fang, "Consumer Demand Analysis of Smart Home Service Market of Internet of Things", Chongqing University of Post and Telecommunications, (2012), p. 30.

[4] K. Lian, "Intelligent multi-sensor control system based on innovative technology integration via ZigBee and Wi-Fi networks", Elsevier, (2013), p. 2.

[5] X. L. Wu, "Principle of Zigbee Wireless Network", (2011), pp. 31-33.

[6] K. Du, "Human machine interactive system on smart home of IoT", Elsevier, (2013).

[7] B. Li, "Research and Application on the Smart Home Based on Component Technologies and Internet of Things", Elsevier, (2011).

[8] G. S. Song, "Comparison Between Bluetooth and Other Short-Range Wireless Communication Technologies", Radio Engineering, (2004), p. 30.

[9] J. Peng, "Research and Design of Bluetooth Home Control Network", Journal of Dalian University of Technology, (2006), p. 1.

[10] L. Guang, "Smart Home System Design Based on Zigbee and Ethernet", Journal of Dalian University of Technology, (2012), p. 1.

[11] X. Q. Wang, "Design and Realization of Zigbee Wireless Sensor Network", (2012), pp. 10-12.

[12] L. Chen, “An Evaluation Study of Mobility Support in ZigBee Networks”, Springer, (2010), p. 3.

[13] L. Kai, "Design and Realization of ZigBee Based Smart Home Control Node", Journal of University of Electronic Science and Technology of China, (2013), p. 1.

[14] Y. Lei, "Design of ZigBee Based Smart Home Monitoring and Controlling System", Journal of Beijing Jiaotong University, (2012), p. 3.

[15] S. Lim, "NBP: light-weight Narrow Band Protection for ZigBee and Wi-Fi coexistence", Springer, (2013), p. 1.

[16] D. Uckelmann, “Architecting the Internet of Things", (2013), pp. 23-40 
International Journal of Smart Home

Vol. 9, No. 1 (2015) 